\title{
An Economic Model of a Professional Sports League
}

\section{Mohamed El-Hodiri and James Quirk}

\author{
University of Kansas, Lawrence
}

\begin{abstract}
This paper investigates the economic structure of professional sports to determine the extent to which the current operating rules justify exemption of professional sports leagues from some aspects of antitrust statutes. We construct a formal decision-making model incorporating certain fundamental features of the industry. Within the context of the model we find that, under current rules of operation, equalization of playing strengths is generally not consistent with profit maximization by teams. However, we suggest a rule that guarantees convergence to a path of equal playing strengths under decentralized control of teams. As an example we study baseball leagues. Some refinements of the model are introduced, for example, incorporating time lags and training costs that result when teams train their own players.
\end{abstract}

Over the past twenty years, the U.S. Congress has held a number of hearings on various bills designed to exempt certain practices of the professional sports industry from prosecution under the federal antitrust statutes. While two bills providing such exemption for special aspects of sports have passed the Congress, one dealing with the AFL-NFL merger in professional football and the second dealing with television and radio contract pooling, the issue of exemption for other phases of professional sports still has not been resolved. It is the purpose of this paper to investigate the economic structure of professional sports to determine the extent to which the current operating rules of professional sports justify such an exemption. Thus this paper considers some of the problems investigated by Rottenberg (1956) in his excellent study of the labor market in baseball, but from a somewhat different point of view and with emphasis on somewhat different aspects of professional sports. The approach adopted here is to

This work was supported in part under grants from the National Science Foundation and the University of Kansas. 
construct a formal decision-making model of a professional sports league, incorporating certain of the more fundamental features peculiar to this industry. Within the context of this model, the issue of "equalization of competitive playing strengths," the basic justification for antitrust exemption, is investigated. It is shown that, under current rules of operation, such equalization is generally not consistent with profit maximization by teams in a professional sports league. A simple rule is suggested, however, which does guarantee convergence to a position of equalization of playing strengths within the context of decentralized control of teams.

\section{2}

Congressional activities relative to professional sports are the result of a series of confusing and, to the layman, contradictory decisions by the U.S. Supreme Court concerning the antitrust status of the various professional sports. Briefly, in 1922 in Baltimore Federal Baseball Club v. National League, a unanimous court held that organized baseball was exempt from operation of the federal antitrust statutes because the business of baseball did not involve an article of commerce in the sense of the commerce clause of the Constitution. This exemption was upheld by a divided court in the 1953 case of Toolson v. New York Yankees. Without examining the facts in this case, the majority argued that stare decisis should apply, particularly in view of the large investments in baseball properties that had been made following the earlier decision. However, in Radovitch v. National Football League, in 1957, the court held that, despite the obvious similarities between the economic structure and rules of professional football and baseball, football was not exempt from application of the antitrust statutes. In the course of that decision, the court stated that, if baseball were to come before the court for the first time again, it would clearly be ruled subject to the antitrust statutes as well. Recognizing the anomaly of this situation, the court requested the Congress to write appropriate legislation to resolve the conflicting treatments afforded basball and the other professional sports. Consequently, the Congress has been attempting, since the early 1950s, to develop and pass a comprehensive bill covering the four major professional team sports of baseball, football, basketball, and hockey. It might be noted parenthetically that it is only because of an almost unbelievable lack of public tact on the part of the owners and controllers of professional baseball that such a bill has not yet been passed. Thus such events as the moves of the New York and Brooklyn franchises to San Francisco and Los Angeles caused tabling of a bill in 1958; later a bill which had passed the House and was sponsored by forty-nine senators was set aside in the wake of rumors concerning a move of the Washington franchise, and similar results followed from the moves of the Milwaukee and Kansas City franchises and the sale of the New York Yankees to the CBS television network. 
More recently, the transfer of the Seattle franchise has again raised the issue of removing baseball's antitrust exemption.

The need for legislation arises from the practices employed by professional sports, many of which would constitute illegal restraints of trade in almost any other industry. These practices are not generally enforceable in the courts but instead are maintained through blacklists and/or other sanctions within the industry. Aside from specific details which differ among the four major professional team sports, these practices relate to (1) rules governing the ownership and acquisition of player contracts, (2) rules governing territorial rights, and (3) rules governing television and radio contracts.

In each of the major professional sports leagues, playing contracts contain a "reserve clause" which effectively binds the player to the team owning the contract for the length of the player's career or until the contract is sold (the "option" clause of football contracts adds some slight flexibility to this). In addition, each of the sports has rules governing the acquisition of new playing talent that typically permit the drafting of new talent in reverse order of finish by teams in the previous year's league standings; the bonus paid to such players is the result of negotiations between the drafting team and the player. There are also rules designed to limit the ability of any team to hold players in minor leagues, and limits on the number of players that any one team may control.

Territorial rights are granted by the league to the teams in the league and typically give exclusive rights to organize a team within a designated geographic area, varying from a 35 - to a 75 -mile radius about the playing field of the team. Fiinally, a bill passed in 1961 grants exemption from antitrust prosecution for contracts signed between professional sports leagues and television or radio networks to control broadcasts or telecasts of league games, with joint participation of league members in the proceeds of such contracts.

Professional sports have requested antitrust exemption for the above practices on the grounds that such practices are necessary to bring about "equalization of playing strengths among teams" and to maintain public confidence in the honesty of the contests. Thus it is argued that, if the reserve clause were eliminated, players would sign contracts with the wealthiest team in the league, thus destroying competition and eventually the sport itself. Further, with players jumping from team to team, the public would begin to suspect players of throwing contests. These arguments about the reserve clause have been dealt with by Rottenberg, who points out that the possibility of buying and selling contracts among teams raises the same problem of acquisition of the best talent by the wealthiest team; however, players will be added by any team only if the marginal revenue product of the player exceeds his cost, so that the dependence of revenue on competition in playing strengths among teams 
makes it unprofitable for any one team to be "too" superior to other teams, whatever the wealth of the team. ${ }^{1}$ Rottenberg shows that the primary impact of the reserve clause is instead a redistribution of income among players and between players and owners.

We are not primarily concerned here with either the equity or other such aspects of the reserve clause, however. Instead, we are interested in the question of whether the collection of rules governing the business aspects of professional sports has any tendency toward equalization of playing strengths among clubs or is in fact consistent with such equalization.

\section{3}

We consider a simplified model of an $n$-team professional sports league which incorporates certain features of the reserve clause, drafting procedures, and provisions governing the sales of player contracts among teams similar to those employed in professional sports. In this section, we ignore the problems associated with minor leagues and the training of players by assuming that newly drafted players add immediately to the stock of playing skills of a team. In section 4 we include the training costs and time lags incident to minor league operations. Thus the model of this section is more applicable to sports such as professional football and basketball, while the model of the next section adds the further complications associated with baseball and hockey.

Each team is assumed to act so as to maximize the discounted present value of net cash flows from operations of the team. We also postulate a perfect capital market so that the rate of discount for each team is the market rate of interest. With each team is associated a stock of "playing skills" which may be altered through purchase of contracts from other teams in the league or through purchase of contracts of new players in the annual draft. In addition, it is assumed that the stock of playing skills for any team depreciates over time at an exponential rate that is the same for every team in the league.

The notion of a stock of playing skills utilized in this paper represents an aggregation over the many specialized and complementary skills required in a professional sports team. In a more detailed treatment, a vector-valued concept incorporating the various specialized skills used by sports teams might be employed, but, because we concentrate on the financial aspects of sports, the aggregated concept appears adequate for our purposes. In the treatment employed here, each player possesses a certain number of units of playing skills, and the strength of a team is

1 Actually, one of the problems Rottenberg does not discuss is how to determine marginal revenue product and marginal cost in professional sports where difficulties arise because of the externalities inherent in the industry (see section 3 ). 
measured simply by summing the playing skills of the players whose contracts are owned by the team. It should be mentioned that, because of this method of aggregation, trades of players for players are not considered in our treatment, but instead only sales of contracts for cash.

The essential economic fact concerning professional team sports is that gate receipts depend crucially on the uncertainty of outcome of the games played within the league. As the probability of either team winning approaches 1 , gate receipts fall substantially. Consequently, every team has an economic motive for not becoming "too" superior in playing talent compared with other teams in the league. On the other hand, gate receipts of the home team are an increasing function of the probability of the home team winning for some range beyond a probability of .5 , so that every team also has an economic motive to be somewhat superior to the rest of the league. Finally, gate receipts at the home park of team $i$ are also a function of the size of the market area in which team $i$ is located.

We formalize the above discussion in terms of the following notation: let

$I_{i}=$ stock of playing skills of team $i$, for $i=1, \ldots, n$;

$x_{i j}=$ number of units of playing skills acquired by team $i$ through purchase of playing contracts of team $j$;

$x_{i N}=$ number of units of playing skills acquired by team $i$ in the draft of new players;

$w_{i}=$ wage rate per unit of playing skills per period paid by team $i$;

$b_{i j}=$ price of a unit of playing skills acquired in a purchase by team $i$ from team $j$;

$b_{i N}=$ price of a unit of playing skills acquired in the draft by team $i$;

$R_{i j}{ }^{(i)}=$ net gate receipts from games played between teams $i$ and $j$ at the home park of team $i$;

$\alpha=$ share of gate receipts paid to the home team;

$M_{i}=I^{i} / \Sigma_{j} I_{j}=$ relative share of playing skills in the league under contract to team $i$;

$P_{i j}=I_{i} /\left(I_{i}+I_{j}\right)=$ probability that team $i$ will defeat team $j$.

Then $R_{i}$ is given by

$$
R_{i}=\alpha \sum_{j \neq i} R_{i j}^{(i)}+(1-\alpha) \sum_{j \neq i} R_{i j}^{(j)},
$$

where $R_{i j}(i)$ is a concave function of $P_{i j}$, attaining a regular maximum at some $P_{i j}^{*}>.5$. We assume a simplified version of the drafting procedure, with $x_{i N}=\mathrm{g}\left(M_{i}\right) x_{N}^{S}$ where $x_{N}^{S}$ is the supply of new playing skills and $g\left(M_{i}\right)$ satisfies $g\left(M_{i}\right)>0$ for $0 \leqslant M_{i}<1$ and $g^{\prime}\left(M_{i}\right)<0$ over this 
range. Further, $\sum_{i} g\left(M_{i}\right)=1$ for $\sum_{i} M_{i}=1$. In particular, $g\left(M_{i}\right)$ can be represented by $g=(n-1)^{-1}\left(1-M_{i}\right)$.

We view the market for existing player contracts as one in which $b_{i j}=$ $b_{i}$ for all $j$, where $b_{i}=b_{\mathrm{i}}\left(\sum_{j} x_{i j}\right), b_{i}^{\prime} \geqslant 0$. That is, for any one team, the price paid for existing players is the same wherever acquired, and the price is a nondecreasing function of the number of playing units demanded. We take the wage rate $w_{i}=w$ for all teams and assume, in addition, $b_{i N}=b_{N}$ for all teams, these prices being independent of inventories and number of new playing units drafted, respectively.

Under these assumptions, the decision problem for team $i$ is the following:

$$
\max \int_{0}^{\infty}\left(R_{i}-w I_{i}-b_{N} x_{i N}-b_{i} \sum_{j} x_{i j}\right) e^{-\delta t}
$$

subject to

$$
\begin{gathered}
I_{j}=x_{j N}+\sum_{k} x_{j k}-\mu I_{j}, \quad j=1, \ldots, n, \\
I_{j} \geqslant 0, \quad j=1, \ldots, n,
\end{gathered}
$$

where $\quad x_{i N}=g\left(M_{i}\right) x_{N}^{S}$ and $x_{i i}=0$ for $i=1, \ldots, n$.

The unique nature of the economic structure of the professional sports industry arises from the fact that team $i$ 's revenues as well as its access to new players depend not only on its own decision variables (particularly $I_{i}$ ) but also on the decision variables of the other teams in the league. Team $i$ 's choice of contract sales $x_{i j}$ partially determines inventories of playing skills for other teams, at least in equilibrium when such choices are consistent with the choices of other teams so that $x_{i j}=-x_{j i}$ for $j \neq i$. However, team $i$ has no control over contract sales among other teams. The consequence is that team $i$ is involved in a problem of decision making under uncertainty involving complex game theoretic difficulties. The joint decision problem for teams in the league may be characterized as an $n$-person nonzero sum differential game, and, as is well known, even the concept of a "solution" to the problem is not well defined. To motivate the approach we will follow, suppose we consider a situation in which equalization of playing strengths characterizes the league, that is, $I_{j}(t)=$ $I_{i}(t)$ for all $i, j=1, \ldots, n$ and all $t$. Then $I_{j}=I_{i}$ is an identity in $t$, so that

$$
x_{j N}+\sum_{k} x_{j k}-\mu I_{j}=x_{i N}+\sum_{k} x_{i k}-\mu I_{i},
$$

$i, j$, and $t$, equalization of playing strength implies $\sum_{k} x_{j k}(t)=\sum_{k} x_{i k}(t)$ for all $i, j$, and $t$. 
If choices of contract sales are mutually consistent among teams, then $x_{i k}=-x_{k i}$ for all $i, k$; hence $\sum_{i} \sum_{k} x_{i k}=0$, which, together with $\sum_{k} x_{j k}=$ $\sum_{k} x_{i k}$, implies $\sum_{k} x_{j k}=0$ for every $j$.

Thus if we restrict our attention to time paths along which equalization of playing strengths occurs, net contract sales by each team in the league are zero, assuming market clearing.

The approach we adopt in this paper is to assume that the firm treats its own contract sales as under its own control, while it assumes that no contract sales take place among other teams. These assumptions are consistent with the profit-maximizing choices of other teams if the league is on a time path of equal playing strengths for all teams and the optimal amount of contract sales $x_{j k}=0$ for every $j, k$. Note that this is somewhat stronger than the assumption of zero net contract sales by each team which is implied by equal playing strengths, but there appears to be only a slight loss of generality in this approach.

The $i$ th team thus chooses optimal time paths $\stackrel{o}{I_{j}^{(i)}},{ }_{i j}^{o}$ for $j=1, \ldots, n$, to maximize the discounted present value of net cash flows, under the assumption that $x_{j k}=0$, for $j, k \neq i$. An equilibrium for the league occurs when the following consistency conditions are met at each point in time:

$$
\begin{gathered}
\stackrel{o}{I_{j}^{(i)}}=\stackrel{o}{I}_{j}^{(k)} \text { for all } i, j, k, \\
x_{i j}^{o}=-x_{j i}^{o}(=0) \quad \text { for all } i, j, \\
\sum_{i=1}^{n} x_{i N}=x_{N}^{S}, \\
b_{i}(0)=b_{j}(0)=b \quad \text { for all } i, j .
\end{gathered}
$$

The profit-maximizing conditions for the $i$ th team are derived as follows: let

$$
\begin{aligned}
H^{i}=\left(R_{i}-w I_{i}-b_{N} x_{i N}-b_{i} \sum_{j=1}^{n} x_{i j}\right) & e^{-\delta t} \\
& +\sum_{j=1}^{n} q_{i j}\left(x_{j N}+\sum_{k=1}^{n} x_{j k}-\alpha_{i} I_{j}\right)
\end{aligned}
$$

be the Hamiltonian for team $i$, where $q_{i j}$ is the multiplier associated with the differential equation for $I_{j}$. At an interior maximizing solution $\left(I_{j}>0\right.$ for every $j$ ), the following conditions hold:

$$
\frac{\partial H^{i}}{\partial x_{i j}}=-\left(b_{i}+x_{i j} b_{i}^{\prime}\right) e^{-\delta t}-q_{i j}+q_{i i}=0, \quad j \neq i,
$$




$$
\begin{aligned}
q_{i j}=-\frac{\partial H^{\imath}}{\partial I_{j}}=- & {\left[\left(\frac{\partial R_{i}}{\partial I_{j}}-w \frac{\partial I_{i}}{\partial I_{j}}-b_{N} \frac{\partial x_{i N}}{\partial I_{j}}\right) e^{-\delta t}\right.} \\
& \left.+\sum_{r=1}^{n} q_{i r}\left(\frac{\partial x_{r N}}{\partial I_{j}}-\mu \frac{\partial I_{r}}{\partial I_{j}}\right)\right], \quad j=1, \ldots, n,
\end{aligned}
$$

$$
I_{j}=x_{j N}+\sum_{k=1}^{n} x_{j k}-\mu I_{j}, \quad j=1, \ldots, n,
$$

$$
\lim _{t \rightarrow \infty} q_{i j}(t)=0, \quad j=1, \ldots, n .
$$

Since $x_{i j}=0$ for every $i, j$, and $b_{i}(0)=b_{j}(0)=b$ for every $i, j$, from condition (i) we have $q_{i j}=q_{i k}$ for $j, k \neq i$; hence denote $q_{j j}$ by $q$ for $j \neq{ }_{i}$.

The variable $q$ may be interpreted as the addition to the discounted present value of cash flows for team $i$ of a unit increase in $I_{j}(j \neq i)$, while $q_{i i}$ is similarly the addition to the discounted present value of cash flows for team $i$ of a unit increase in $I_{i}$.

We first consider the question of consistency of profit-maximizing choices by teams with a time path characterized by equal playing strengths, in the special case where $b_{i}$ is treated as a parameter by team $i$, so that $b_{i}^{\prime}=0$. The basic proposition that holds is the following:

Proposition 1.-If $b_{i}$ is treated as a parameter by the $i$ th team profit maximization by each team is inconsistent with a time path of equal playing strengths for all teams.

In essence, as is easily verified, this result follows from the "bang-bang" nature of the maximization problem when $b_{i}$ is a parameter, since $x_{i j}$ enters both the objective function and the constraints in linear fashion. If $x_{i j}$ is completely unconstrained in magnitude, a profit-maximizing position does not exist; if $x_{i j}$ is restrained by a (finite) positive upper bound and negative lower bound, $x_{i j}=0$ does not occur at a profit-maximizing position.

When team $i$ regards $b_{i}$ as a strictly increasing function of $\Sigma x_{i j}$, then along a path playing strengths condition (ii) may be rewritten as

$$
\begin{aligned}
& \dot{q}=-\left[\left(\frac{\partial R_{i}}{\partial I_{j}}-b_{N} \frac{\partial x_{i N}}{\partial I_{j}}\right) e^{-\delta t}\right. \\
& \left.+q\left(\sum_{r \neq 1} \frac{\partial x_{r N}}{\partial I_{j}}-\mu\right)+q_{i i} \frac{\partial x_{i N}}{\partial I_{j}}\right], j \neq i, \\
& \dot{q}_{i i}=-\left[\left(\frac{\partial R_{i}}{\partial I_{i}}-w-b_{N} \frac{\partial x_{i N}}{\partial I_{i}}\right) e^{-\delta t}\right.
\end{aligned}
$$




$$
\left.+q \sum_{r \neq i} \frac{\partial x_{r N}}{\partial I_{i}}+q_{i i}\left(\frac{\partial x_{i N}}{\partial I_{i}}-\mu\right)\right]
$$

Further,

$$
\frac{\partial x_{r N}}{\partial I_{j}}=\stackrel{s}{x_{N} g^{\prime}}\left(-\frac{I_{r}}{I_{T}^{2}}\right), \quad j \neq r,
$$

while

$$
\frac{\partial x_{r N}}{\partial I_{r}}=x_{N}^{s} g^{\prime}\left(\frac{I_{T}-I_{r}}{I_{T}^{2}}\right),
$$

where $I_{T}=\sum_{j=1}^{n} I_{j}$. With equal playing strengths for all teams, $M_{r}=1 / n$ for every $r$; hence $\partial x_{r N}=-\left(1 / I n^{2}\right) x_{N}^{S} g^{\prime}$ for $j \neq r$, and $\partial x_{r N} / \partial I_{r}=$ $\left[(n-1) / I n^{2}\right] x_{N}^{S} g^{\prime}$, where $g^{\prime}$ is evaluated at $M_{r}=1 / n$ for every $r$, and $I_{j}=I$ for $j=1, \ldots, n$.

Thus (ii') can be simplified to

$$
\begin{gathered}
\dot{q}=-\left[\frac{\partial R_{i}}{\partial I_{j}} e^{-\delta t}+b_{N} \frac{x_{N}^{S}}{I n^{2}} g^{\prime} e^{-\delta t}\right. \\
\left.+q\left(\frac{x_{N}^{S} g^{\prime}}{I n^{2}}-\mu\right)-q_{i i} \frac{x_{N}^{S} g^{\prime}}{I n^{2}}\right], j \neq i, \\
\dot{q}_{i i}=-\left[\left(\frac{\partial R_{i}}{\partial I_{i}}-w\right) e^{-\delta t}-b_{N} \frac{x_{N}^{S}(n-1) g^{\prime}}{I n^{2}} e^{-\delta t}\right. \\
\left.-q \frac{x_{N}^{S}(n-1) g^{\prime}}{I n^{2}}+q_{i i}\left(\frac{x_{N}^{S}(n-1) g^{\prime}}{I n^{2}}-\mu\right)\right] .
\end{gathered}
$$

From condition (ii) we immediately obtain proposition 2 .

Proposition 2.-Profit maximizing is consistent with equal playing strengths only if $\partial R_{i} / \partial I_{j}=\partial R_{i} / \partial I_{k}$ for all $j, k \neq i$ and for all $i$.

In effect, proposition 2 asserts that the market areas in which teams are located must not differ from one another sufficiently so that team $i$ finds its gate receipts affected more by an increase in the strength of one team than by an increase in the strength of another, given that all teams are of equal strength.

To examine further the properties of the solution time path under equal playing strengths, introduce the following notation and change of variables:

Let $\partial R_{i} / \partial I_{j}=c$ for $j \neq i$, and let $\partial R_{i} / \partial I_{i}-w=d$. Further, let $Z=$ $q e^{\delta t}$ and $y=q_{i i} e^{\delta t}$. Then 


$$
\begin{aligned}
& Z=a_{11} Z+a_{12} y+a_{10}, \\
& \dot{y}=a_{21} Z+a_{22} y+a_{20},
\end{aligned}
$$

where

$$
\begin{aligned}
& a_{11}=\delta+\mu-\frac{x_{N}^{s} g^{\prime}}{I n^{2}}, \\
& a_{12}=\frac{g^{\prime} x_{N}^{s}}{I n^{2}}, \\
& a_{10}=-c-b_{N} \frac{x_{N}^{s} g^{\prime}}{I^{2}}, \\
& a_{21}=\frac{x_{N}^{\mathrm{s}}(n-1) g^{\prime}}{I n^{2}}, \\
& a_{22}=\delta+\mu-\frac{x_{N}^{\mathrm{s}}(n-1) g^{\prime}}{\operatorname{In}^{2}}, \\
& a_{20}=-d+b_{N} \frac{x_{N}(n-1) g^{\prime}}{\operatorname{In}^{2}} .
\end{aligned}
$$

Choose $\bar{Z}$ and $\bar{y}$ to satisfy $\dot{z}=0$ and $\dot{y}=0$. Then $\bar{Z} \bar{y}$ are uniquely determined if and only if $a_{11} a_{22}-a_{12} a_{21} \neq 0$. But $a_{11} a_{22}-a_{12} a_{21}=$ $(\delta+\mu)\left(\delta+\mu-x_{N}^{S} g^{\prime} /\right.$ In $)>0$, since $\delta>0, \mu>0, g^{\prime}<0$. Hence

$$
\begin{aligned}
& \bar{Z}=\frac{1}{(\delta+\mu)\left(\delta+\mu-\mu g^{\prime} / g\right)}\{c(\delta+\mu) \\
&\left.-\frac{\mu g^{\prime}}{g n}\left[c(n-1)+d-b_{N}(\delta+\mu)\right]\right\}, \\
& \bar{y}=\frac{1}{(\delta+\mu)\left(\delta+\mu-\mu g^{\prime} / g\right)} {\left[(d-c)(\delta+\mu)-\frac{\mu g^{\prime}}{g} b_{N}(\delta+\mu)\right], }
\end{aligned}
$$

from which it follows that

$$
\frac{\partial R_{i}}{\partial I_{i}}-\frac{\partial R_{i}}{\partial I_{j}}=(\delta+\mu) b+\frac{\mu g^{\prime}}{g}\left(b_{N}-b\right)+w .
$$

It might further be noted that, since 


$$
\begin{gathered}
R_{i}=\alpha \sum_{j \neq i} R_{i j}(i)+(1-\alpha) \sum_{j \neq i} R_{i j}(j), \\
\frac{\partial R_{i}}{\partial I_{i}}=\frac{1}{4 I}\left\{\sum_{j \neq i}\left[\alpha \frac{\partial R_{i j}(i)}{\partial p_{i j}}+(1-\alpha) \frac{\partial R_{i j}(j)}{\partial p_{i j}}\right]\right\},
\end{gathered}
$$

and

$$
\frac{\partial R_{i}}{\partial I_{k}}=-\frac{1}{4 I}\left[\alpha \frac{\partial R_{i k}(i)}{\partial p_{i k}}+(1-\alpha) \frac{\partial R_{i k}(k)}{\partial p_{i k}}\right], \quad k \neq i,
$$

along a time path of equal playing strengths.

Since each of the $n$ teams in the league satisfies the relations above, we have

$$
\frac{\partial R_{i}}{\partial I_{j}}=\frac{\partial R_{i}}{\partial I_{k}}, \frac{\partial R_{j}}{\partial I_{i}}=\frac{\partial R_{j}}{\partial I_{k}}, \frac{\partial R_{k}}{\partial I_{i}}=\frac{\partial R_{k}}{\partial I_{j}}, \quad i \neq j \neq k .
$$

Further, $\partial R_{i k^{(i)}} / \partial p_{i k}=-\left(\partial R_{i k}^{(i)} / \partial p_{k i}\right)$, so that the following relations hold among teams (where $\partial R_{i k}{ }^{(i)} / \partial p_{i k}$ is denoted by $a_{i k}^{i}$ ):

$$
\begin{aligned}
& \alpha\left(a_{i k}^{i}+a_{k i}^{k}-a_{i j}^{i}-a_{j i}^{j}\right)=a_{k i}^{k}-a_{j i}^{j}, \\
& \alpha\left(a_{k i}^{k}+a_{i k}^{i}-a_{k j}^{k}-a_{j k}^{j}\right)=a_{i k}^{i}-a_{j k}^{j}, \\
& \alpha\left(a_{j i}^{j}+a_{i j}^{i}-a_{j k}^{j}-a_{k j}^{k}\right)=a_{i j}^{i}-a_{k j}^{k} .
\end{aligned}
$$

In particular, these relations are satisfied when all teams have identical revenue functions so that, evaluated at $p_{i j}=.5$ for all $i \neq j$, we may denote $\mathrm{a}_{i j}^{i}$ by $\theta$ for all $i \neq j$. By our earlier discussion, $\theta>0$ (home gate receipts are an increasing function of the probability of winning for some range beyond a probability of winning of .5). In this special case, one may establish that the home team's share of gate receipts must exceed .5 .

Proposition 3.-If all revenue functions of teams in the league are identical, a time path along which playing strengths are equal is consistent with profit maximization by teams only if $\alpha$, the home team's share of gate receipts, exceeds .5 .

To prove this proposition, note that

$$
\frac{\partial R_{i}}{\partial I_{i}}-\frac{\partial R_{i}}{\partial I_{j}}=\frac{n}{4 I}(2 \alpha-1) \theta=(\delta+\mu) b+\frac{\mu g^{\prime}}{g}\left(b_{N}-b\right)+w,
$$

so that

$$
\alpha=.5+\frac{2 I}{\theta n}\left[(\delta+\mu) b+\frac{\mu g^{\prime}}{g}\left(b_{N}-b\right)+w\right]>.5,
$$


since $\theta>0, g^{\prime} / g<0$, and $b_{N} \leqslant b$.

Intuitively, this result holds because the expressions for $\partial R_{i} / \partial I_{i}$ and $\partial R_{i} / \partial I_{k}$ are given by

$$
\begin{aligned}
& \frac{\partial R_{i}}{\partial I_{i}}=\frac{1}{4 I}(n-1)(2 \alpha-1) \theta, \\
& \frac{\partial R_{i}}{\partial I_{k}}=-\frac{1}{4 I}(2 \alpha-1) \theta, \quad k \neq i .
\end{aligned}
$$

If $\alpha=.5$ revenue for team $i$ is a maximum (with respect to its own inventory) at a position of equal playing strengths, and the marginal revenue for team $i$ is the same from adding a unit to its own inventory as selling a unit to any other team. But adding a unit to its own inventory involves the additional costs of (1) paying out $b$ dollars for such a unit, (2) losing the draft preference by being stronger than other teams, and (3) paying wages of $w$ dollars per unit purchased. All of these costs are reflected in the term $(\partial+\mu) b+\left(\mu g^{\prime} / g\right)\left(b_{N}-b\right)+w$. Hence profit maximization is inconsistent with $\alpha=.5$ and also for $\alpha<.5$.

To summarize, we have shown that a time path along which playing strengths are equal for all teams is consistent with profit maximization by teams only if (1) net sales of contracts by every team are zero, (2) the purchase price of existing player contracts is not treated parametrically by teams, (3) revenue functions for all teams are similar in the sense that $\partial R_{i} / \partial I_{j}=\partial R_{i} / \partial I_{k}$ for every $i \neq j \neq k$; (4) if revenue functions are identical for all teams, in which case $\alpha>.5$.

The most important point concerning this analysis is that, even with the reserve clause acting as a constraint on the movement of players to the wealthier teams and even with the draft system operating to equalize strengths, the rules permitting sales of player contracts among teams lead to unequal playing strengths so long as franchises are located in cities with markedly different revenue potentials. It is not surprising, then, that casual empiricism indicates that in no professional team sport under current rules of operation is there a tendency toward equal playing strengths.

The histories of the various professional sports contain numerous instances of attempts to alter rules of operation to offset the tendency toward dominance of a league by its wealthier teams, including the introduction of the reserve clause, drafting procedures, limitations on player rosters, restrictions on ownership of farm clubs, and even attempts to syndicate a sport under common ownership. All of these have thus far failed in their objectives, because of the imbalance of revenue potential among franchises. Under the conditions characterizing the model of this paper, however, there is a simple change in the rules of professional sports leagues that guarantees convergence to a state of equal playing strengths, given only that the supply of new playing skills is constant over time. This is summarized in proposition 4. 
Proposition 4.-Assume that the supply of new playing skills is constant over time. If sales of player contracts for cash are forbidden, then, given any distribution of initial stocks of playing skills among teams, time paths of stocks of playing skills converge to one of equal playing strengths for all teams.

To prove proposition 4 , recall that the basic differential equation governing the stock of playing skills for team $i$ is given by

$$
\dot{I}_{i}=x_{i N}+\sum_{j} x_{i j}-\mu I_{i}, \quad i=1, \ldots, n .
$$

If sales of contracts are forbidden, $x_{i j}=0$ for every $i, j$; hence $\dot{I}_{i}=x_{i N}-$ $\mu I_{i}$, where

$$
x_{i N}=\frac{1}{n-1}\left(1-\frac{I_{i}}{I_{T}}\right) x_{N}^{S}, \quad \text { where } \quad I_{T}=\sum_{j=1}^{n} I_{j} .
$$

Then $\dot{I}_{T}=x_{N}^{S}-\mu I_{T}$, where $x_{N}^{S}$ is a constant independent of $t$. Solving this differential equation we obtain

$$
I_{T}=\left[I_{T}(0)-\frac{x_{N}^{S}}{\mu}\right] e^{-\mu t}+\frac{x_{N}^{S}}{\mu},
$$

where $I_{T}(0)$ is the initial stock of playing skills in the league.

Let $v=I_{i}-I_{j}$ so that $\dot{v}=\dot{I}_{i}-\dot{I}_{j}$; hence

$$
\begin{aligned}
& \dot{v}=\left(x_{i N}-x_{j N}\right)-\mu\left(I_{i}-I_{j}\right) \\
& \therefore \dot{v}=-\left[\frac{x_{N}^{S}}{(n-1) I_{T}}+\mu\right] v,
\end{aligned}
$$

and

$$
\frac{d v}{v}=-\left[\mu+\alpha\left(\frac{1}{\beta e^{-\mu t}+\gamma}\right)\right] d t
$$

where

$$
\alpha=\frac{x_{N}^{s}}{n-1}, \beta=\left(I_{T}(0)-\frac{x_{N}^{S}}{\mu}\right), \gamma=\frac{x_{N}^{S}}{\mu} .
$$

Hence $\ln v=-\mu t-\alpha \int d t /\left(\beta e^{-\mu t}+\gamma\right.$.

Let $y=\beta e^{-\mu t}+\gamma$ so that $d y=-\mu \beta e^{-\mu t} d t$. Thus 


$$
\begin{aligned}
\int \frac{d t}{\beta e^{-\mu t}+\gamma} & =-\frac{1}{\mu}\left[\int \frac{d y}{-\gamma y}+\int \frac{d y}{\gamma(y-\gamma)}\right] \\
& =-\frac{1}{\mu}\left[-\frac{\ln y}{\gamma}+\frac{1}{\gamma} \ln (y-\gamma)\right] .
\end{aligned}
$$

Hence

$$
v=K\left(\frac{y-\gamma}{y}\right)^{a / \gamma \mu} e^{-\mu t}
$$

where

$$
K=v(0)\left(\frac{\beta+\gamma}{\beta}\right)^{a / \gamma \mu}
$$

After substitution we obtain

$$
I_{i}(t)-I_{j}(t) \stackrel{\left[I_{i}(0)-I_{j}(0)\right]\left\{I_{T}(0)^{1 /(n-1)} e^{[-\mu t n /(n-1)]}\right\}}{\left[\left(I_{T}(0)-x_{N}^{S} / \mu\right) e^{-\mu t}+x_{N}^{S} / \mu\right]^{1 /(n-1)}} .
$$

Thus if $I_{T} \neq 0$ for any $t, \lim _{t \rightarrow \infty} I_{i}(t)-I_{j}(t)=0$, and hence convergence to a state of equal playing strengths characterizes the league.

From the proof of proposition 4, in particular the expression for $I_{i}(t)-$ $I_{j}(t)$, we obtain an immediate corollary.

Proposition 5.-If the supply of new playing talent is constant over time, then (with cash sales of contracts forbidden) convergence to a state of equal playing strengths occurs more rapidly the fewer teams there are in the league and the larger the rate of depreciation of playing skills is.

To illustrate, under a set of rules banning the sales of player contracts for cash, one would expect to find a more rapid convergence to a position of equal playing strengths in football than in baseball because the playing lifetime in football is considerably less than in baseball, and the number of teams participating in the annual player draft is roughly the same in the two sports twenty-four in baseball, twenty-six in football). Proposition 5 also indicates that, in general, expansion of leagues through the addition of franchises tends to operate in the direction of offsetting convergence.

The fundamental reason that convergence to equal playing strengths occurs when sales of contracts are banned is that this effectively eliminates the operation of the profit motive by owners in dealings among teams in the league, although such a motive may still be exercised in the internal operations of the team itself. Because of the externalities inherent in professional sports, profit maximization does not necessarily lead to desirable, much less optimal, results even from the point of view of the owners of the teams and, as indicated above, generally conflicts with the 
equalization of strengths in the league that represents the primary justification for antitrust exemption for team sports.

These conclusions may be extended to the case where training of players occurs as an activity of teams in the league. Section 4 deals with this case.

\section{4}

In baseball and hockey, new players acquired in the draft typically require an extended period of training in the minor leagues before they are skillful enough to be of value to the major league team. In this section we consider the decision problem facing owners of teams when time lags and training costs are incorporated into the problem. The notation employed above is used in this section as well, and the basic assumptions made concerning the earlier model apply to this model.

Let $Z_{i}(t)$ denote the stock of playing skills in training at time $t$ and let the training period be $\tau$ units of time. Then

$$
\dot{Z}_{i}(t)=x_{i N}(t)-x_{i N}(t-\tau) .
$$

We assume that the cost of training is an increasing function of the stock of playing skills in the process of training; hence this cost may be written as a function $f_{i}\left(Z_{i}\right)$, where $f_{i}^{\prime}>0$.

Firm $i$ acts to solve the following problem:

$$
\max \int_{0}^{\infty}\left[R_{i}-w I_{i}-b_{i} \sum_{j} x_{i j}-b_{N} x_{i N}-f_{i}\left(Z_{i}\right)\right] e^{-\delta t},
$$

subject to

$$
\begin{aligned}
& \dot{I}_{j}(t)=x_{j N}(t-\tau)+\sum_{k} x_{j k}(t)-\mu I_{j}(t), \quad j=1, \ldots, n, \\
& \dot{Z}_{i}(t)=x_{i N}(t)-x_{i N}(t-\tau) .
\end{aligned}
$$

As in the earlier analysis, equalization of playing strengths implies zero net trades by all teams. We adopt the stronger assumption of zero trades among all teams.

Assuming that $x_{j k}=0$ for $j, k \neq i$, and assuming that $b_{i}$ is an increasing function of $\Sigma x_{i j}$, necessary conditions for a maximum as given by Halany (1968) are the following: let

$$
H^{i}=\pi_{i} e^{-\delta t}+\sum_{j} q_{i j} I_{j}+p_{i} \dot{Z}_{i}
$$

where

$$
\pi_{i}=R_{i}-w I_{i}-b_{i} \sum_{j} x_{i j}-b_{N} x_{i N}-f_{i}\left(Z_{i}\right) .
$$

Then at a maximum,

$$
\frac{\partial H^{i}}{\partial x_{i j}}=\left(-b_{i}-x_{i j} b_{i}^{\prime}\right) e^{-\delta t}+q_{i i}-q_{i j}=0, \quad j=1, \ldots, n,
$$




$$
\begin{aligned}
\dot{p}_{i} & =-\frac{\partial H^{i}}{\partial Z_{i}}=\dot{f}_{i}^{\prime} e^{-\delta t} \\
\dot{q}_{i i} & =-\frac{\partial H^{i}}{\partial I_{i}}=-\left\{\left(\frac{\partial R_{i}}{\partial I_{i}}-w-b_{N} \frac{\partial x_{i N}}{\partial I_{i}}\right) e^{-\delta t}\right. \\
& \left.+\sum_{r} q_{i r}(t+\tau) \frac{\partial x_{r N}}{\partial I_{i}}-q_{i i} \mu+\frac{\partial x_{i N}}{\partial I_{i}}[p(t)-p(t+\tau)]\right\} \\
\dot{q}_{i j}= & -\frac{\partial H^{i}}{\partial I_{j}}=-\left\{\left(\frac{\partial R_{i}}{\partial I_{j}}-b_{N} \frac{\partial x_{i N}}{\partial I_{j}}\right) e^{-\delta t}\right. \\
& \left.+\sum_{r} q_{i r}(t+\tau) \frac{\partial x_{r N}}{\partial I_{j}}-q_{i j} \mu+\frac{\partial x_{i N}}{\partial I_{j}}[p(t)-p(t+\tau)]\right\} \\
& j \neq i, \\
\dot{I}_{j}(t)= & x_{j N}(t-\tau)+\sum_{k} x_{j k}(t)-\mu I_{j}(t), \quad j=1, \ldots, n, \\
\dot{Z}_{i}(t)=x_{i N}(t)-x_{i N}(t-\tau), & \lim _{t \rightarrow \infty} q_{i j}(t)=0, \quad j=1, \ldots, n,
\end{aligned}
$$

Consider now an equilibrium time path along which $x_{i j}=0$ for every $i, j$ and all teams have equal playing strengths. Assume in addition that the stock of new playing skills is constant over time. Then $x_{i N}=x_{j N}=x_{N}$ is a constant for all $i, j$; hence

$$
Z_{i}=\int_{t-\tau}^{t} x_{i N}=\tau x_{N}
$$

is a constant, and $\dot{p}_{i}=f_{i}^{\prime} e^{-\delta t}$ may be solved to obtain $p_{\imath}=-\left[\left(e^{-\delta t} / \delta\right) \times\right.$ $\left.f^{\prime}\left(\tau x_{N}\right)\right]$. (By transversality condition $\lim _{t \rightarrow \infty} p_{\imath}(t)=0$; hence $p_{i}(0)=-$ $\left.\left(f^{\prime} / \delta\right)\left(\tau x_{N}\right)\right)$.

From condition (5), $q_{i j}=q_{i k}$ for all $j, k \neq i$; hence denote $q_{i j}$ by $q$ for $j \neq i$. It is immediately clear that proposition 1 of section 1 holds for this model as well, since $x_{i j}$ enters linearly into the objective function and the constraints. Hence $b_{i}$ cannot be treated parametrically by any team if equal playing strengths and profit maximization occur.

The expressions for $\dot{q}_{i i}$ and $\dot{q}$ can be simplified to 


$$
\begin{gathered}
\dot{q}=-\left\{\frac{\partial R_{i}}{\partial I_{j}} e^{-\delta t}+b_{N} \frac{x_{N}^{S}}{I n^{2}} g^{\prime} e^{-\delta t}+q(t+\tau) \frac{x_{N}^{S} g^{\prime}}{I n^{2}}-q(t) \mu\right. \\
\left.-q_{i i}(t+\tau) \frac{x_{N}^{S} g^{\prime}}{I n^{2}}-\frac{f^{\prime}\left(\tau x_{N}\right)}{\delta} \frac{x_{N}^{S} g^{\prime}}{I n^{2}} \cdot\left[-e^{-\delta t}-e^{-\delta(t-\tau)}\right]\right\}, j \neq i \\
\dot{q}_{i i}=-\left\{\left(\frac{\partial R_{i}}{\partial I_{i}}-w\right) e^{-\delta t}-b_{N} \frac{x_{N}^{S}(n-1)}{I n^{2}} g^{\prime} e^{-\delta t}\right. \\
-q(t+\tau) \frac{x_{N}^{S}(n-1) g^{\prime}}{I n^{2}}+q_{i i}(t+\tau) \frac{x_{N}^{S}(n-1) g^{\prime}}{I n^{2}}-q_{i i}(t) \mu \\
\left.+\frac{f^{\prime}\left(\tau x_{N}\right)}{\delta} \frac{(n-1)}{I n^{2}} x_{N}^{S} g^{\prime}\left[-e^{-\delta t}-e^{-\delta(t-\tau)}\right]\right\} .
\end{gathered}
$$

Thus proposition 2 holds as well, since $\partial R_{i} / \partial I_{j}=\partial R_{i} / \partial I_{k}$ for all $j$, $k \neq i$. Because of the complexities associated with obtaining explicit solutions to mixed difference-differential equation systems, no attempt has been made to verify proposition 3 , however.

It remains to consider the problem of convergence to a position of equal playing strengths from an arbitrary initial distribution among teams of stocks of playing skills, when sales of playing contracts are banned. From the condition $x_{i j}=0$ for all $i, j$ we obtain $I_{i}(t)=x_{i N}(t-\tau)-\mu I_{i}$ for $i=1, \ldots, n$. Assume $x_{N}^{S}$ is a constant over time. Then

$$
\dot{I}_{T}=x_{N}^{s}-\mu I_{T} \quad \text { where } \quad I_{T}=\sum_{j=1}^{n} I_{j} ;
$$

hence

$$
I_{T}(t)=\left[I_{T}(0)-\frac{x_{N}^{S}}{\mu}\right] e^{-\mu t}+\frac{x_{N}^{S}}{\mu} .
$$

Let $v(t)=I_{i}(t)-I_{j}(t)$ :

$$
\begin{aligned}
\dot{v}(t) & =\left[x_{i N}(t-\tau)-x_{j N}(t-\tau)\right]-\mu v(t) \\
& =-\frac{x_{N}}{n-1}\left[\frac{v(t-\tau)}{I_{T}(t-\tau)}\right]-\mu v(t) \\
\therefore \dot{v}(t) & =-\frac{-x_{N}^{S} v(t-\tau)}{(n-1)\left\{\left[I_{T}(0)-x_{N}^{s} / \mu\right] e^{-\mu(t-\tau)}+x_{N}^{S} / \mu\right\}}-\mu v(t) .
\end{aligned}
$$

To show that $\lim _{t \rightarrow \infty} v(t)=0$, we use the following result of El'sgol'tc (1966, p. 72, example 1): 
Theorem. $-\dot{x}+a x(t)+b(t) x(t-\tau)=0$ is (asymptotically) stable if $|b(t)|<a$ for all $t$.

Let $a=\mu$ and

$$
b(t)=\frac{x_{N}^{S}}{(n-1)\left\{\left[I_{T}(0)-x_{N}^{S} / \mu\right] e^{-\mu t} e^{\mu \tau}+x_{N}^{S} / \mu\right\} .}
$$

Let $l(t)=\left[I_{T}(0)-x_{\Lambda}^{S} / \mu\right] e^{-\mu t} e^{\mu \tau}$, so that $l^{\prime}(t)=-\mu l(t)$. If $I_{T}(0)=$ $x_{N}^{S} / \mu$, then $b(t)=\mu / \mathrm{n}-1$; hence $|b(t)|<a=\mu$. If $I_{T}(0)>x_{N}^{S} / \mu$, then $\max _{t}|b(t)|=b(0)$. But

$$
b(0)=\frac{x_{N}^{S}}{(n-1)}\left\{\left[I_{T}(0)-x_{N}^{S} / \mu\right] e^{\mu \tau}+x_{N}^{S} / \mu\right\}^{-1}<\frac{\mu}{n-1}<a .
$$

If $I_{T}(0)<x_{N}^{S} / \mu$ and $I_{T}(t)>0$ for all $t$, then $\sup _{t}|b(t)|=\lim _{t \rightarrow \infty} b(t)$. But $\lim _{t \rightarrow \infty} b(t)=\mu /(n-1)<a$; hence convergence occurs for arbitrary initial conditions.

Thus propositions 1, 2, and 4 hold when training of players is incorporated into the decision problem of professional sports teams.

\section{5}

The general conclusion that one may derive from the analysis of the paper is that, under present rules of operation, professional sports leagues will exhibit no tendency toward equalization of playing strengths. If franchises were located in areas with generally equal revenue potential, equalization might occur even under the present rules, but this condition is patently violated in all of the four major professional sports. On the other hand, changing the rules to prohibit the sales of player contracts among teams guarantees convergence over time to equal playing strengths. Such a rule would generally violate the antitrust statutes, since it can only be promulgated and enforced by joint action of all teams in the league. However, antitrust exemption to permit such a provision appears to be amply justified because of the externalities peculiar to professional sports. On the other hand, the current antitrust exemption afforded professional baseball has not resulted in institutional changes in the industry leading toward equalization of playing strengths.

\section{References}

El'sgol'tc, L. E. Introduction to the Theory of Differential Equations with Deviating Arguments. Moscow, 1964. English trans. San Francisco, Calif.: Holden-Day, 1966.

Halanay, A. "Optimal Controls for Systems with Time Lag." SIAM J. on Control (June 1968), pp. 215-34.

Rottenberg, S. "The Baseball Players Labor Market." J.P.E. 64 (June 1956): 256. 
Copyright of Journal of Political Economy is the property of University of Chicago Press and its content may not be copied or emailed to multiple sites or posted to a listserv without the copyright holder's express written permission. However, users may print, download, or email articles for individual use. 